\title{
Diffuse Interstitial Pulmonary Infiltrates in Malignant Melanoma
}

\author{
Jens Bräunlich MD, Hans-Jürgen Seyfarth MD, Armin Frille MD, and Hubert Wirtz MD
}

\begin{abstract}
Only a few cases of sarcoidosis-like reaction to a pharmacologic compound have been reported in patients with melanoma in the literature. Long-term treatment with interferon alpha may be assumed. We report a case of a 25 -y-old man who presented to our department with diffuse interstitial pulmonary infiltrates and slightly enlarged mediastinal lymph nodes as determined by computed tomography. He had a history of malignant melanoma with 18 months of interferon alpha-2 therapy. Histological analysis of a transbronchial biopsy revealed sarcoidosis-like reactions. Key words: sarcoidosis-like reactions; melanoma; rare lung diseases; drug reactions; interferon alpha-2. [Respir Care 2015;60(7):e115-e117. (c) 2015 Daedalus Enterprises]
\end{abstract}

\section{Introduction}

A sarcoidosis-like reaction is a rare diagnosis in melanoma patients who are treated with interferon alpha. Differential diagnosis includes metastatic and infectious infiltrations. Morphological signs of sarcoidosis-like reaction usually regress spontaneously.

\section{Case Report}

A 25-y-old man presented to our department with diffuse interstitial pulmonary infiltrates and slightly enlarged mediastinal lymph nodes as determined by computed tomography (Fig. 1). The computed tomography scan was initially ordered for staging after discovery of an enlarged cervical lymph node, with a history of malignant melanoma of the left retroauricular region excised 5 y previously in 2008 (pT2bN0M0, Clark level IV, Breslow thickness of $1.4 \mathrm{~mm}$ ). Surgery at that time included parotidectomy and sentinel lymph node biopsy, both negative for a metastatic tumor. Following surgery, interferon alpha-2 therapy $(3 \times 3$ million IU subcutaneously per

The authors are affiliated with the Department of Pulmonary Medicine, University of Leipzig, Leipzig, Germany.

The authors have disclosed no conflicts of interest.

Correspondence: Jens Bräunlich MD, Department of Pulmonary Medicine, University of Leipzig, Liebigstrasse 20, 04103 Leipzig, Germany. E-mail: highflow@web.de.

DOI: $10.4187 /$ respcare. 03621

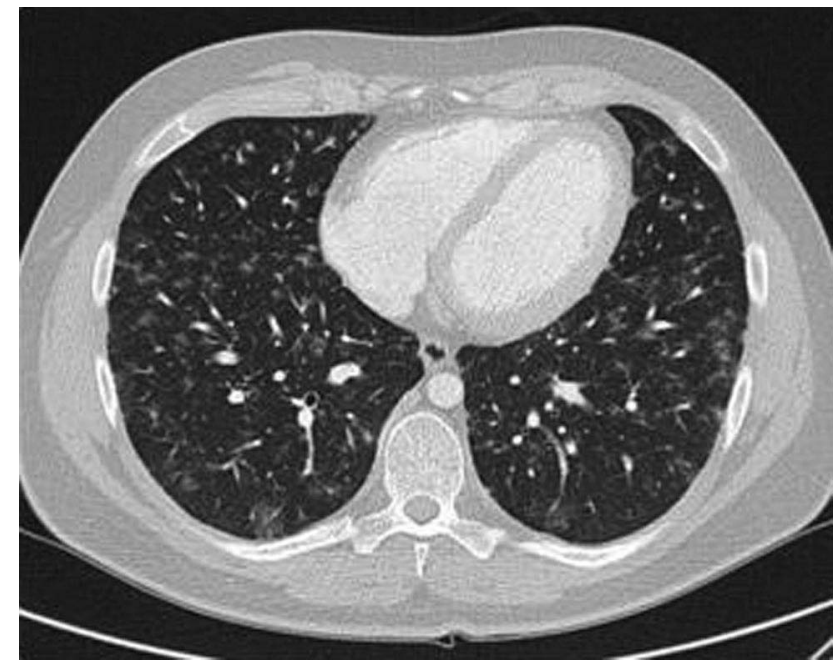

Fig. 1. Computed tomography scan of the thorax with illustration of diffuse interstitial pulmonary infiltrates in all lobes of the lungs and slightly enlarged mediastinal lymph nodes showing no proof of metastasis due to malignant melanoma.

week) was initiated for 18 months. At the time of the computed tomography scan, the patient complained of a nonproductive morning cough, but no fever, sweating, or weight loss. There was no conspicuous travel history, and the patient had ongoing nicotine abuse of 10 pack-years. A positron emission tomography scan revealed 3 cervical lymph nodes as well as pulmonary infiltrates exhibiting increased glucose metabolism (Figs. 2 and 3). Atypical pneumonia was suspected, but in view of the melanoma history, a recurrence of this disease was not excluded. 


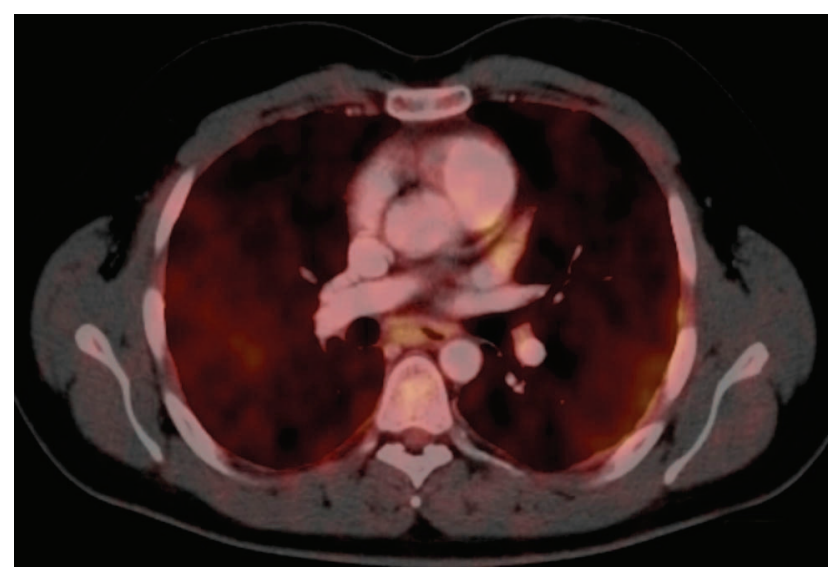

Fig. 2. Positron emission tomography-computed tomography scan depicts areas with active glucose metabolism in both lungs after bronchoalveolar lavage and transbronchial biopsy.

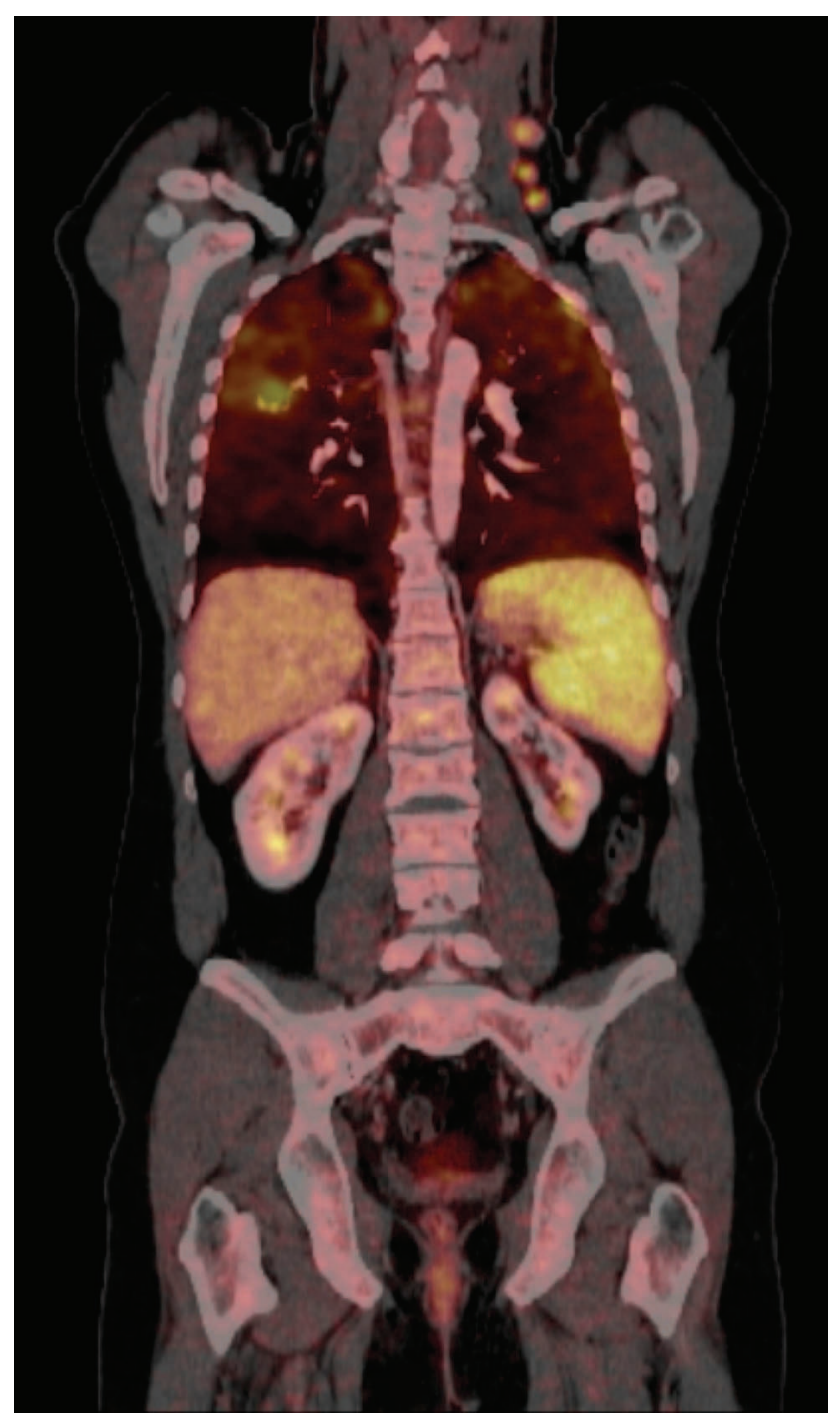

Fig. 3. Positron emission tomography-computed tomography scan illustrates 3 left-sided cervical lymph nodes (levels 4 and 5) with increased glucose metabolism.
Serology and induced sputum culture were without any evidence of respiratory infection. The serum levels of the soluble interleukin-2 receptor $(1,230 \mathrm{U} / \mathrm{mL})$, angiotensinconverting enzyme $(72 \mathrm{U} / \mathrm{L})$, and neopterin $(11 \mathrm{nmol} / \mathrm{L})$ were markedly increased. Bronchoalveolar fluid cell analysis showed low-level lymphocytosis (15\%) with an increased CD4/CD8 ratio (5.0). Histologic analysis of bronchial and transbronchial biopsies revealed numerous CD68-positive macrophages and epithelioid/giant-cell granulomas, but no Melan-A-positive or HMB-45-positive tumor cells (Figs. 4 and 5).

The patient was diagnosed with a sarcoidosis-like reaction most likely caused by interferon therapy. Unfortunately, the subsequently resected cervical lymph nodes showed metastatic melanoma in addition to sarcoid-like lesions.
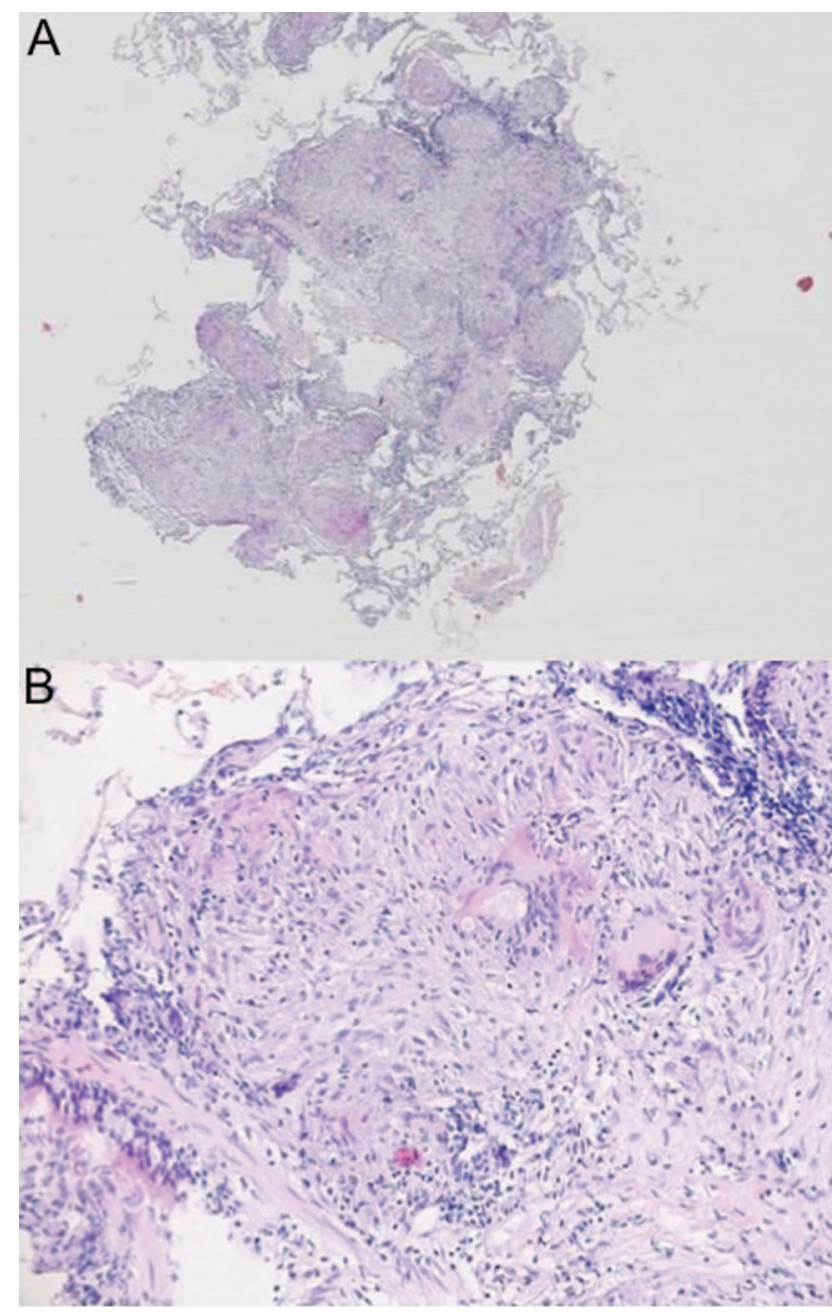

Fig. 4. Hematoxylin-and-eosin stain of a transbronchial biopsy from the apical segment of the right lower lobe showing many subepithelial, partly confluent, epithelioid/giant-cell granulomas and chronic inflammation. A: $\times 5$ objective. B: $\times 20$ objective. 


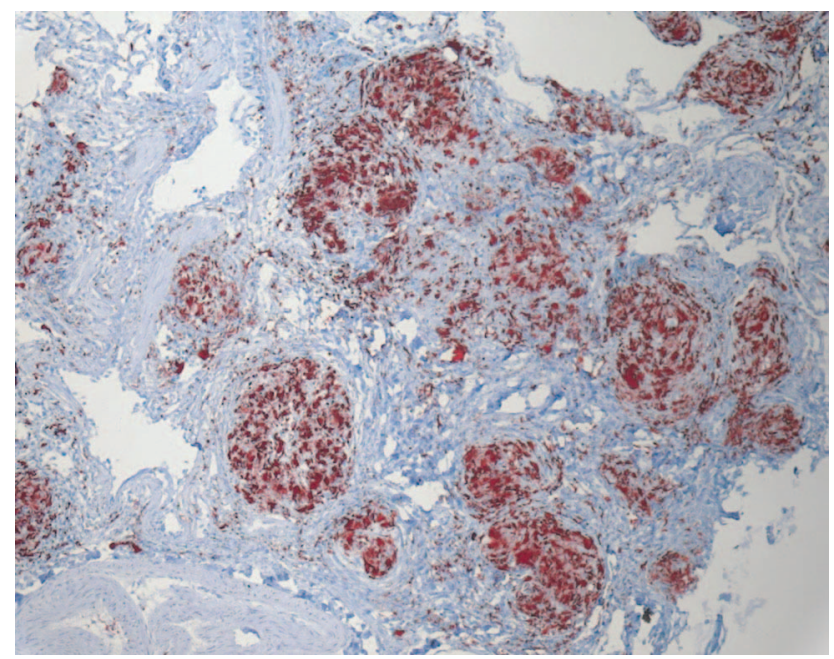

Fig. 5. Immunohistochemical stain of CD68 antigen to identify histiocytes forming epithelioid/giant-cell granulomas (transbronchial biopsy from the apical segment of the right lower lobe).

\section{Discussion}

Only a few cases of sarcoidosis-like reactions to a pharmacologic compound in patients with melanoma have been reported in the literature. ${ }^{1}$ Long-term treatment with interferon alpha may be assumed. ${ }^{2}$

Interferons are pleiotropic cytokines that exhibit antiviral, antiproliferative, antitumor, and immunomodulatory effects. This anticancer effect is used in therapy for melanoma, lymphoma, and leukemia. ${ }^{3}$ The interaction between interferon alpha and interleukin-2 is poorly understood, yet a previous study supports the hypothesis that the interferon-induced $\mathrm{T}$ helper 1 cytokine response plays a pivotal role in the development of granulomatous inflamma- tion. ${ }^{4}$ Following $\mathrm{T}$ cell recognition of antigens, $\mathrm{CD} 4{ }^{+} \mathrm{T}$ cells secrete $\mathrm{T}$ helper 1-type cytokines such as interferons, interleukin-2, and tumor necrosis factor alpha. The secretion of interferons and tumor necrosis factor alpha promotes macrophage accumulation, activation, and aggregation, leading to granulomatous inflammation.

The time frame from the start of therapy to the granulomatous reaction varies from a few months up to $3 \mathrm{y} .^{2}$ Very rarely, a diagnosis is made only after the completion of therapy. Skin, lung, and mediastinal lymph nodes are mostly involved, but manifestations of other organ systems can occur. Usually, the morphological signs of this sarcoidosis-like tissue reaction regress spontaneously after cessation of interferon therapy. As for this case report, the time period until the sarcoidosis-like reaction resolves may be beyond the observation period.

In summary, a sarcoidosis-like reaction is a rare but important differential diagnosis in melanoma patients who are treated with interferon alpha and can mimic metastatic infiltrations. In our case, both the long time period between initiation of interferon therapy and onset of symptoms and the lack of spontaneous regression after therapy cessation are unusual.

\section{REFERENCES}

1. Massaguer S, Sánchez M, Castel T. Mediastinal sarcoidosis induced by high-dose alpha-2-interferon therapy in a patient with malignant melanoma. Eur Radiol 2004;14(9):1716-1717.

2. North J, Mully T. Alpha-interferon induced sarcoidosis mimicking metastatic melanoma. J Cutan Pathol 2011;38(7):585-589.

3. Borden EC. Review: Milstein Award lecture: interferons and cancer: where from here? J Interferon Cytokine Res 2005;25(9):511-527.

4. Chen ES, Moller DR. Sarcoidosis-scientific progress and clinical challenges. Nat Rev Rheumatol 2011;7(8):457-467. 